\title{
Human oncoprotein MDM2 activates the Akt signaling pathway through an interaction with the repressor element-1 silencing transcription factor conferring a survival advantage to cancer cells
}

\author{
S Singh ${ }^{1,3}$, M Ramamoorthy ${ }^{1,3,4}$, C Vaughan ${ }^{1}$, WA Yeudall ${ }^{2}$, S Deb $^{1}$ and S Palit Deb ${ }^{*, 1}$
}

The current paradigm states that the Akt signaling pathway phosphorylates the human oncoprotein mouse double minute 2 (MDM2), leading to its nuclear translocation and degradation of the tumor suppressor p53. Here we report a novel Akt signaling pathway elicited by MDM2. Upregulation of endogenous MDM2 promotes, whereas its downregulation diminishes, Akt phosphorylation irrespective of p53 status. MDM2 requires phosphatidylinositol (PI)3-kinase activity for enhancing Akt phosphorylation and upregulates this activity by repressing transcription of the regulatory subunit p85 of PI3-kinase. MDM2 interacts with the repressor element-1 silencing transcription factor (REST), a tumor suppressor that functions by downregulating PI3-kinase activity and Akt phosphorylation, prevents localization of REST on the p85 promoter and represses p85 expression. The deletion mutant of MDM2 capable of upregulating Akt phosphorylation represses p85 expression and interferes with localization of REST on the p85 promoter, whereas the deletion mutant of MDM2 that does not increase Akt phosphorylation cannot perform these functions. Silencing of REST abrogates the ability of MDM2 to upregulate Akt phosphorylation and downregulate p85 expression, implicating the ability of MDM2 to interact with REST in its ability to inhibit p85 expression and activate Akt phosphorylation. Inhibition of MDM2-mediated Akt phosphorylation with an Aktphosphorylation-specific inhibitor abrogates its ability to improve cell survival. Consistently, the Akt phosphorylation function of MDM2 was required for its ability to improve cell survival after treatment with a chemotherapeutic drug. Our report not only unravels a novel signaling pathway that contributes to cell survival but also implicates a p53-independent transcription regulatory function of MDM2 in Akt signaling.

Cell Death and Differentiation (2013) 20, 558-566; doi:10.1038/cdd.2012.153; published online 14 December 2012

The human homolog of the mouse double minute-2 gene product (MDM2) is frequently overexpressed in human sarcomas and carcinomas, and amplification of the mdm2 gene enhances the tumorigenic potential of murine cells, ${ }^{1,2}$ implicating the genetic alteration in oncogenesis. MDM2 interacts with several growth suppressors, including the wild-type (WT) tumor suppressor p53, the retinoblastoma susceptibility gene product $(\mathrm{Rb})$ and the growth suppressor p14. ${ }^{1,3}$ Although these interactions contribute to its oncogenic function, overexpression of MDM2 is thought to induce oncogenesis primarily by inactivating WT p53.

MDM2 recognizes the transactivation domain of p53 and inactivates p53-mediated transcriptional activation; ${ }^{4}$ it is also an
E3 ubiquitin ligase and degrades p53 by targeting it for ubiquitination. ${ }^{5}$ Small-molecule anti-MDM2 drugs, such as Nutlin or MDM2-specific silencer RNA, induce apoptosis and drug sensitivity in cell lines harboring WT p53 by elevating p53 levels. ${ }^{6}$ Overexpression of MDM2 reduces the chemotherapeutic sensitivity of cancer cells by inactivating and degrading WT p53. ${ }^{7}$

Cancer cells with mutated p53 often overexpress MDM2, and the prognosis of this group of cancers is worse than their WT p53-containing counterparts. ${ }^{2}$ Although WT p53-dependent oncogenic functions of the oncoprotein are well studied, the significance of its WT p53-independent oncogenic functions $^{1-3}$ in normal or cancer cell biology remains underexplored.

${ }^{1}$ Goodwin Research Laboratory, Department of Biochemistry and Molecular Biology, The Massey Cancer Center, Virginia Commonwealth University, Richmond, VA, USA and ${ }^{2}$ The Philips Institute of Oral and Craniofacial Molecular Biology, Virginia Commonwealth University, Richmond, VA, USA

*Corresponding author: S Palit Deb, Goodwin Research Laboratory, Department of Biochemistry and Molecular Biology, The Massey Cancer Center, Virginia Commonwealth University, PO Box 980035, 401 College Street, Richmond, VA 23298, USA. Tel: 804828 9541; Fax: 804827 1427; E-mail: spdeb@vcu.edu

${ }^{3}$ These authors contributed equally to this work.

${ }^{4}$ Current address: Department of Pathology, Kimmel Center for Biology and Medicine of the Skirball Institute, New York University School of Medicine, New York, NY, USA

Keywords: MDM2; Akt phosphorylation; Akt signaling; REST; p85

Abbreviations: MDM2, mouse double minute 2 protein; Akt, serine-threonine specific protein kinase B; p-Akt, phospho-Akt; REST, repressor element-1 silencing transcription factor; PI3-kinase, kinase that phosphorylates hydroxyl group at 3-position of the inositol ring of phosphatidylinositol; PI4-kinase, kinase that phosphorylates hydroxyl group at 4-position of the inositol ring of phosphatidylinositol; PTEN, phosphatase and tensin homolog; QPCR, quantitative polymerase chain reaction; ChIP, chromatin immunoprecipitation; GSK3 $\beta$, glycogen synthase kinase $\beta$; p-GSK3 $\beta$, phospho-GSK3 $\beta$; SH-6, inhibitor of Akt phosphorylation (also known as Akt inhibitor III); Erk2, extracellular signal-regulated kinase 2; shRNA, short hairpin RNA; GFP, green fluorescence protein; CFP, cyan fluorescence protein; YFP, yellow fluorescence protein; DMSO, dimethyl sulfoxide; MEF, mouse embryo fibroblast; mTOR, mammalian target of rapamycin; Rictor, rapamycin-insensitive companion of mTOR. Received 29.2.12; revised 26.9.12; accepted 19.10.12; Edited by M Oren; published online 14.12 .12 
MDM2 interacts with several components of the Akt (serine-threonine specific protein kinase B) signaling pathway. It is phosphorylated at Ser 166 and Ser 186 by the phosphatidylinositol (PI)3-kinase pathway, and interacts with protein kinase B Akt, which phosphorylates MDM2,, ${ }^{8}$ promoting its nuclear entry and degradation. ${ }^{9}$ Apart from its functional interaction with Akt, MDM2 interacts with the translation elongation factor EF1 $\alpha$, which is known to upregulate PI4kinase and to interact with Akt2. ${ }^{10}$

The Akt signaling pathway transmits signals from membrane-bound receptors and regulates cell proliferation, survival and motility, processes that are deregulated during tumorigenesis. ${ }^{11}$ Activation of the pathway has been implicated in diminished sensitivity of cancer cells to chemotherapeutic drugs. ${ }^{11}$ Mutations in genes, such as phosphatase and tensin homolog (PTEN) or repressor element-1 silencing transcription factor (REST), encoding regulators of this pathway have been found in various cancers, and have also been found to be correlated with poor prognosis. ${ }^{12-14}$

As MDM2 interacts with several components of the Akt signaling pathway, and its overexpression has been strongly correlated with oncogenesis as well as survival of cancer cells after treatment with chemotherapeutic drugs, ${ }^{7}$ we sought to determine if MDM2 modulates Akt phosphorylation. Our data show that MDM2 interacts with a regulator of PI3-kinase, REST, inhibits its ability to induce expression of the regulatory subunit of PI3-kinase and enhances PI3-kinase activity, and thus Akt phosphorylation. MDM2-mediated Akt phosphorylation reduces death of cancer cells by chemotherapeutic drugs in the absence of WT p53.

\section{Results}

Upregulation of endogenous MDM2 induces, while its knockdown reduces, Akt phosphorylation. As MDM2 interacts with several components of the Akt signaling pathway, ${ }^{8,10}$ we investigated the ability of endogenous MDM2 to elicit Akt phosphorylation. For this purpose, we generated littermate MDM2 transgenic mice with a p53 + / + and p53-/- background. Quantitative polymerase chain reaction (QPCR) analysis of genomic DNA isolated from these cells showed a 10-fold higher copy number of the $m d m 2$ gene than littermate non-transgenic mice. ${ }^{15}$ Following a method described earlier, we derived cultured lung cells from these mice ${ }^{16}$ and analyzed their extracts for Akt phosphorylation at Ser 473 by western blotting. Our analysis revealed that lung cells derived from at least four sets of MDM2 transgenic mice had five- to sevenfold higher levels of phospho-Akt ( $p$-Akt) compared with lung cells from littermate non-transgenic mice irrespective of their p53 status (Figures $1 \mathrm{a}$ and $\mathrm{b}$ and Supplementary Figures S1A and B). These results indicate that increase in the levels of MDM2 causes increase in Akt phosphorylation.

Knockdown of MDM2 expression in p53-/- MDM2 transgenic cells, using lentiviral vectors described earlier, ${ }^{17}$ reduced Akt phosphorylation (Supplementary Figure S1C), indicating that elevated levels of MDM2 upregulate Akt phosphorylation in p53-/- MDM2 transgenic lung cells. As Akt phosphorylates MDM2, ${ }^{8,9}$ we confirmed that elevation of endogenous levels of MDM2 leads to its phosphorylation by Akt. Furthermore, $\mathrm{SH}-6$, an inhibitor of Akt phosphorylation (also known as Akt inhibitor III), inhibited phosphorylation of MDM2 (Supplementary Figure S2A).

To determine if MDM2-mediated Akt phosphorylation would also lead to phosphorylation of one of its known substrates, glycogen synthase kinase $\beta($ GSK $3 \beta),{ }^{12}$ we determined the levels of phospho-GSK3 $\beta$ (p-GSK3 $\beta$ ) in the lung cells described above by western blot analysis. Our data revealed that lung cells generated from two p53-/-/MDM2 transgenic mice had higher levels of $\mathrm{p}-\mathrm{GSK} 3 \beta$ than lung cells generated from two littermate p53-/ - mice (Figure 1c and Supplementary Figure S1A). An inhibitor of Akt phosphorylation, SH-6, abrogated the increased phosphorylation of GSK3 $\beta$ in the presence of MDM2 (Supplementary Figure $\mathrm{S} 2 \mathrm{~B})$.

We next determined if knockdown of endogenous MDM2 levels would reduce p-Akt levels in cultured tumor-derived cell lines. Three plasmids harboring a puromycin resistance gene were constructed, two of which expressed two different short hairpin RNAs (shRNAs) against MDM2 and one expressed shRNA against a non-endogenous luciferase gene. The plasmids were introduced into the osteosarcoma cell line, OsACL (SJSA-1), which harbors WT p53 and overexpresses MDM $2,{ }^{18}$ by nucleofection. Puromycin-resistant colonies were generated and pooled. Extracts prepared from puromycin-resistant pooled cell colonies were subjected to western blot analysis to determine the levels of MDM2 and phosphorylated Akt. The results (Figure 1d) showed that expression of MDM2 shRNA inhibited MDM2 expression and Akt phosphorylation compared with control luciferase shRNA. These data show that diminished MDM2 expression in OsACL cells reduces Akt phosphorylation, indicating that endogenously overexpressed MDM2 is capable of enhancing Akt phosphorylation.

As knockdown of MDM2 should upregulate WT p53 expression in OsACL cells, we determined if endogenous MDM2 enhances Akt phosphorylation in the presence of mutant p53, and if knockdown of MDM2 in the presence of mutant p53 could diminish Akt phosphorylation. For this experiment, we chose a lung cancer cell line, $A B C 1$, which harbors mutant $p 53 .{ }^{19}$ We infected $A B C 1$ cells with lentiviral vectors expressing shRNA against MDM2 or green fluorescence protein (GFP) as described earlier, ${ }^{17}$ and selected puromycin-resistant colonies to generate $A B C 1$ cell lines with reduced expression of $\mathrm{MDM} 2$, or control $A B C 1$ cells expressing shRNA against GFP. The levels of MDM2 and p-Akt in extracts prepared from the $A B C 1$ constructs were then determined by western blot analysis. Results of this experiment revealed that $A B C 1$ constructs expressing MDM2 shRNA contained reduced levels of MDM2 and p-Akt compared with the extracts expressing control GFP shRNA (Figure 1e), indicating that MDM2 upregulates Akt phosphorylation.

We further investigated if elevation of endogenous MDM2 in the presence of WT p53 would increase Akt phosphorylation. For this purpose, we used a small-molecule inhibitor of MDM2, Nutlin-3, which binds to the p53-binding region of MDM2, which in turn stabilizes p53 and elevates MDM2 


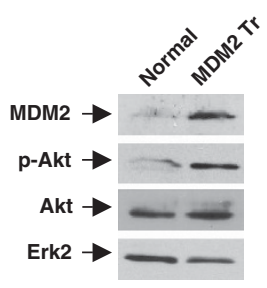

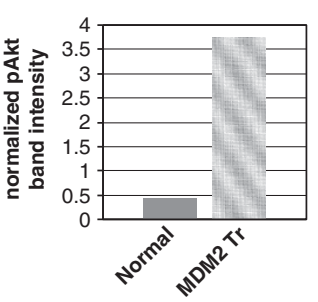

C
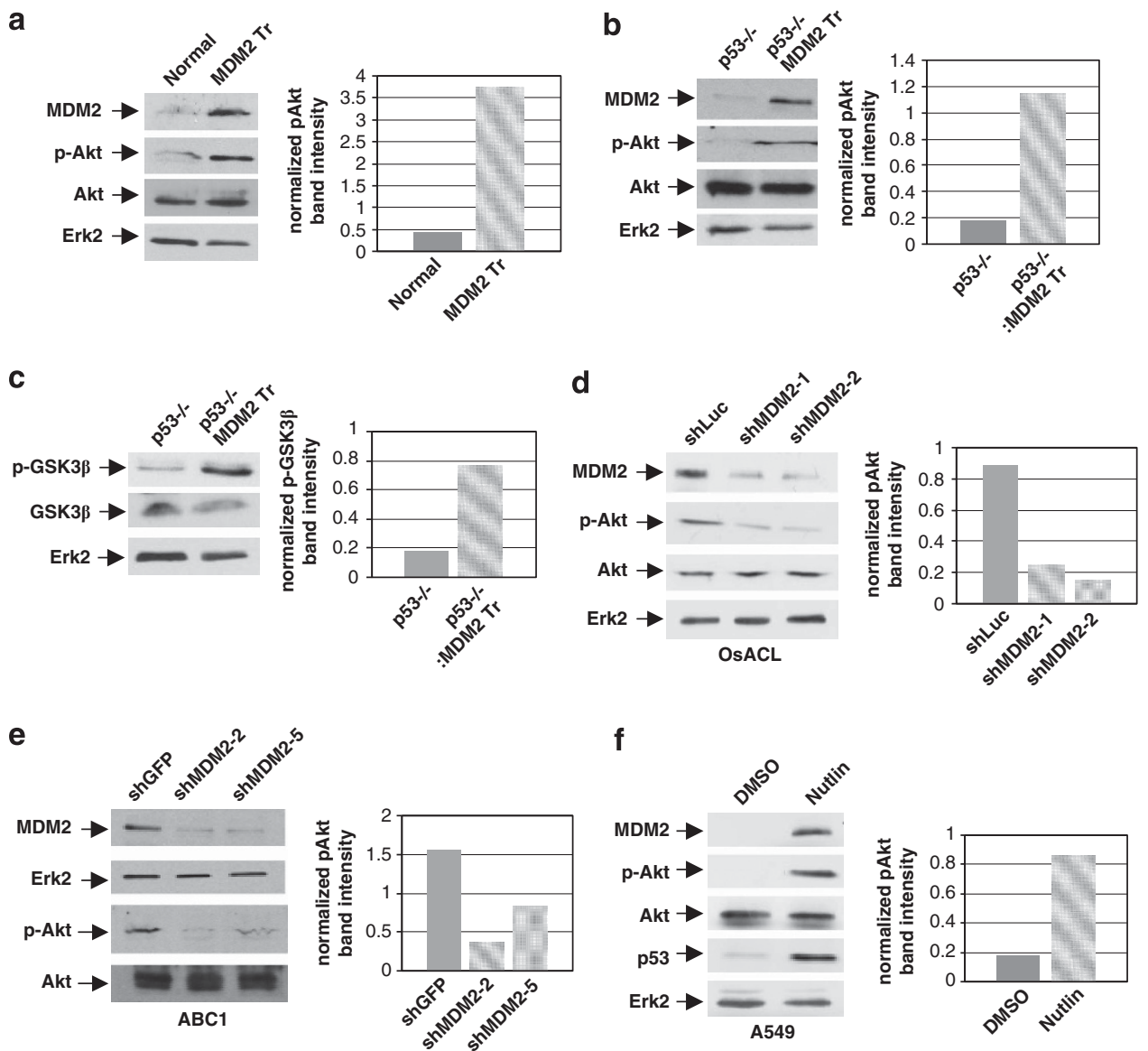

f

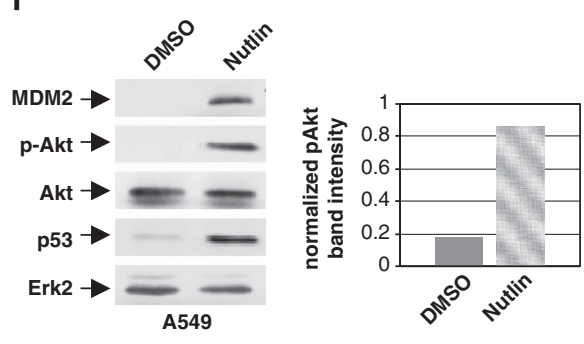

Figure 1 Increase or knockdown of endogenous MDM2 levels increases or reduces Akt phosphorylation. Western blot analysis of extracts prepared from lung cells derived from littermate (a) p53 $+/+$ and p53 $+/+$ MDM2 transgenic (MDM2 Tr), (b, c) p53 - I - and p53 - / - MDM2 transgenic (MDM2 Tr) mice, (d) OsACL cells transfected with plasmids expressing MDM2 shRNA (shMDM2-1 or -2) or luciferase shRNA (shLuc), (e) ABC1 cells stably expressing shRNA against MDM2 (shMDM2-2 or -5) or GFP shRNA (shGFP) and (f) A549 cells treated with Nutlin-3 or vehicle (DMSO). Migration of MDM2, p-Akt, Akt, GSK3 $\beta$ phosphorylated at Ser 9 (p-GSK3 $\beta$ ), GSK3 $\beta$ and Erk2 are indicated. Differences in the p-Akt or p-GSK3 $\beta$ levels are shown by graphs at the right panel of each western blot

expression. ${ }^{6,20}$ A549 lung cancer cells, which harbor WT p53, ${ }^{21}$ were treated with Nutlin-3 or vehicle (dimethyl sulfoxide (DMSO)). Western blot analysis of the cell extracts revealed that treatment of cells with Nutlin-3 upregulated p53 and MDM2 expression as expected and also upregulated p-Akt levels (Figure 1f). This result indicates that elevation of MDM2 levels elevates Akt phosphorylation even in the presence of elevated WTp53. Taken together, these data show that increase or decrease in the levels of MDM2 increase or reduce p-Akt levels, respectively, independent of the p53 status of the cells. Our data also suggest that the p53-interaction domain of MDM2, which interacts with Nutlin-3, is not required for Akt phosphorylation.

Consistent with the above findings, we used an Akt activity reporter $^{22,23}$ to show that absence of p53 or MDM2 in MEF cells downregulates Akt phosphorylation, and this deficiency could be rescued by re-expressing MDM2 alone (Supplementary Figure S3), indicating a requirement of MDM2 in efficient Akt signaling in non-transformed cells. Furthermore, transient or stable ectopic expression of MDM2 in p53-null cells activates Akt phosphorylation at Ser 473 (Supplementary Figure S4).
MDM2 upregulates PI3-kinase activity. As Akt phosphorylation is a downstream event of phosphorylation of phosphatidylinositol bis phosphate (PIP2) to phosphatidylinositol 3,4,5 phosphate (PIP3) by PI3-kinase, we investigated if MDM2 is capable of upregulating PI3-kinase activity. For this experiment, we used a PI3-kinase activity reporter ${ }^{22}$ as described in Materials and Methods. The PI3-kinase activity reporter expresses a protein similar to the Akt activity reporter, but harbors a consensus PI3-kinase substrate peptide sequence in place of the $\mathrm{p}$-Akt substrate peptide sequence (Supplementary Figure S3B).

The PI3-kinase activity reporter was introduced by nucleofection into H1299 cells together with MDM2 expression plasmid or vector plasmid. The nucleofected cells were then fixed and analyzed by confocal imaging as described in the Materials and Methods. As shown in Figure 2a, expression of MDM2 in H1299 cells substantially enhanced phosphorylation of the substrate peptide sequence of the PI3-kinase activity reporter and, therefore, emission of yellow fluorescence in contrast to the introduction of the empty vector plasmid. The increase in the yellow fluorescence protein/cyan fluorescence protein (YFP/CFP) fluorescence ratio over background was 


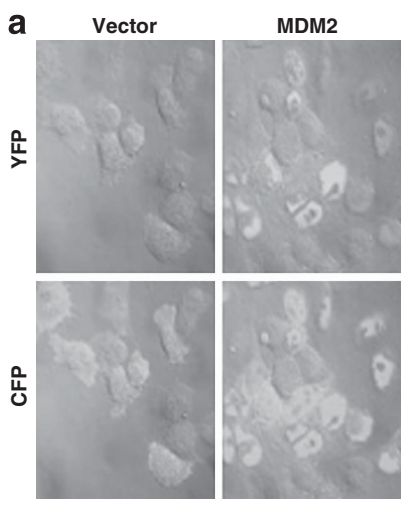

b

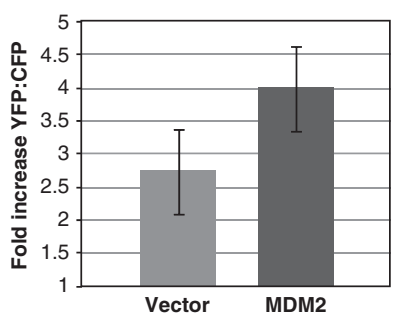

C

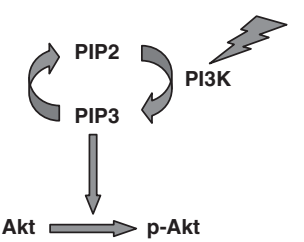

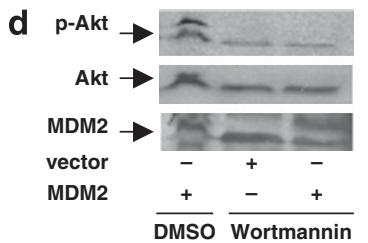

Figure 2 MDM2 upregulates PI3-kinase activity. Endogenous PI3 kinase activity was determined by confocal microscopy after the introduction of a PI3-kinase activity reporter and a plasmid expressing MDM2 in $\mathrm{H} 1299$ cells (a). Increase in the YFP/CFP ratio over background in each case is shown by a bar graph (b). At least 50 cells were analyzed in each case. The cartoon shows PI3kinase activates Akt phosphorylation (c). Treatment of H1299 cells stably expressing MDM2 with wortmannin abrogates MDM2-mediated Akt phosphorylation (d). The figure shows western blot analysis of extracts prepared from $\mathrm{H} 1299$ cells stably expressing MDM2 (MDM2) or stably transfected with vector plasmid (vector) after wortmannin treatment. Migration of the control MDM2, p-Akt and Akt bands are shown by arrows

significantly higher in the presence of MDM2 compared with cells that received the vector plasmid (Figure $2 \mathrm{~b}$ ). The observed increase was found to be as efficient as reported for response of the PI3-kinase activity reporter to various growth factors. ${ }^{22,23}$ These data show that MDM2 is capable of upregulating PI3-kinase activity.

A PI3-kinase inhibitor, wortmannin, inhibits MDM2mediated Akt phosphorylation. To determine if MDM2 requires the PI3-kinase pathway to activate Akt phosphorylation (Figure 2c), MDM2-mediated Akt phosphorylation was analyzed after treatment with wortmannin, which has been shown to inhibit PI3-kinase activity. ${ }^{24}$ H1299 cells stably expressing MDM2, or stably transfected with vector plasmid, were treated with wortmannin or vehicle (DMSO) for $4 \mathrm{~h}$, and levels of MDM2 and p-Akt in the cell extracts were determined by western blot analysis. As shown in Figure 2d, wortmannin inhibited Akt phosphorylation as expected, and cells stably expressing MDM2 failed to rescue Akt phosphorylation inhibited by wortmannin. This observation shows that inhibition of PI3-kinase activity abrogates MDM2-mediated Akt phosphorylation, implicating the requirement of PI3kinase activity for Akt phosphorylation by MDM2.

MDM2 downregulates the expression of p85, the regulatory subunit of PI3-kinase. In the basal state of the PI3kinase, its regulatory subunit p85 interacts with its catalytic subunit p110 through the inter-Src homology 2 domain of p85 and inhibits its catalytic activity. ${ }^{25,26}$ Both the regulatory subunit p85 and the catalytic subunit p110 are often mutated in cancer, leading to the activation of Akt signaling and cell transformation. ${ }^{27,28}$ These mutations are thought to disrupt the inhibitory interaction between p85 and the catalytic subunit. $^{29}$

To decipher the mechanism of enhanced Akt phosphorylation by MDM2, we considered if MDM2 targets the expression of the known negative regulator of PI3-kinase, p85. We therefore determined the expression of p85 in ABC1 lung cancer cell lines expressing shRNA against MDM2 or GFP both at the level of protein using western blot analysis, and RNA using QPCR as described earlier. ${ }^{17}$ Our data revealed that the levels of both p85 protein (Figure 3a) and transcripts (Figure $3 b$ ) were higher in the $A B C 1$ constructs expressing MDM2 shRNA compared with those isolated from ABC1 cells expressing shRNA against GFP. These data show that knockdown of MDM2 increases p85 expression. We further determined if an increase in MDM2 expression would downregulate p85 expression, and therefore determined p85 protein levels in lung cells derived from littermate p53 - / and p53-/-/MDM2 transgenic mice and p53+/+ and p53 + / + /MDM2 transgenic mice by western blot analysis. Our data show that lung cells from p53-/ - mice express approximately threefold higher p85 compared with that of p53 - / - /MDM2 transgenic mice (Figures 3c and d). A similar reduction in p85 expression was observed in lung cells from p53 $+/+/$ MDM2 transgenic mice when compared with that from p53 $+/+$ mice (Figures $3 e$ and f). These data show that MDM2 downregulates p85 expression and suggest that it releases PI3-kinase activity and thus Akt phosphorylation.

MDM2 interacts with REST and interferes with its ability to localize on the p85 promoter. The ability of MDM2 to induce Akt phosphorylation in cell lines lacking PTEN such as OsACL (Figure 1d) or H1299 (Supplementary Figure S4) precluded involvement of PTEN, a known negative regulator of the PI3-kinase, ${ }^{13}$ in the Akt phosphorylation function of MDM2. Akt phosphorylation is also modulated by the rapamycin-insensitive companion of mTOR-mammalian target of rapamycin (Rictor-mTOR) pathway. ${ }^{30}$ However, silencing of Rictor did not prevent MDM2 to enhance Akt phosphorylation (data not shown).

REST restricts PI3-kinase activity and Akt phosphorylation and is frequently mutated in cancer cells. ${ }^{14,31,32}$ Immunoprecipitation of MDM2 with anti-MDM2 antibody co-immunoprecipitated REST in A549 cell extracts (Supplementary Figure S5), suggesting an interaction between the two proteins.

As MDM2 does not reduce REST protein levels (Supplementary Figure S6), we determined if MDM2 inhibits localization of REST on the p85 promoter using chromatin immunoprecipitation (ChIP) assay. H1299 cells stably expressing either MDM2 or its deletion mutant that upregulates Akt phosphorylation (Del 1-120) or the deletion mutant (Del 491155) that lacks this ability (Supplementary Figure S7) were subjected to ChIP analysis using a REST antibody. Quantification of the p85 promoter from the recovered DNA fragments showed that the REST antibody immunoprecipitated the p85 promoter in $\mathrm{H} 1299$ cells containing empty vector, but not appreciably from $\mathrm{H} 1299$ cells expressing either 
a
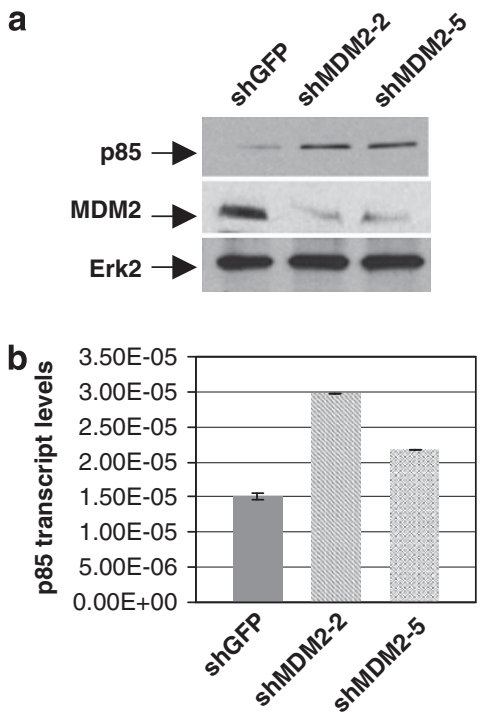

c
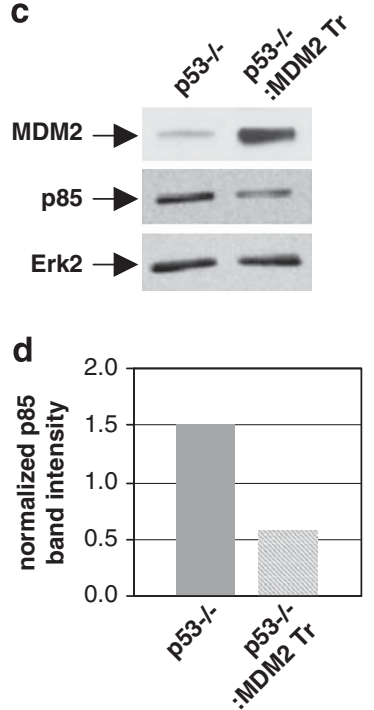

e

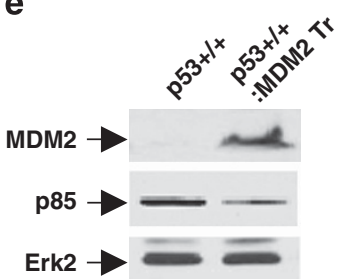

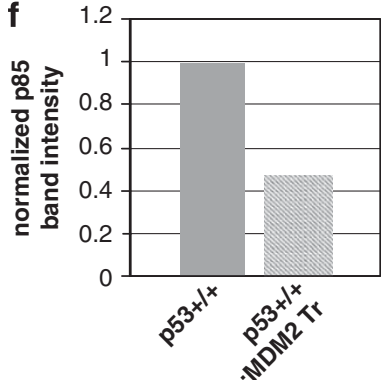

Figure 3 MDM2 levels inversely control p85 expression. $(\mathbf{a}, \mathbf{b})$ Knockdown of endogenous MDM2 levels leads to increase in the expression of the regulatory subunit of PI3-kinase, p85. Endogenous p85 protein (a) and transcript (b) levels in ABC1 cells stably expressing shRNA against MDM2 (shMDM2-2 or -5) or GFP (shGFP) were determined by western blot analysis and QPCR respectively. (c-f) Increase in endogenous MDM2 levels reduces p85 protein expression. Endogenous p85 protein levels in lung cells from littermate p53 - I - and p53 - I - /MDM2 transgenic (c) or p53 $+1+$ and p53 + I+ /MDM2 transgenic (e) mice were determined by western blot analysis. Densitometric analyses of p85 expression in MDM2 transgenic lung cells are shown (d, f). Migration of p85 and Erk2 are indicated. Band intensities were normalized to the levels of Erk2

MDM2 or its deletion mutant Del $1-120$, which is capable of Akt phosphorylation. p85 promoter fragments recovered from these cell extracts were close to that recovered from IgG immunoprecipitates (Figure 4a). On the other hand, the REST antibody co-immunoprecipitated p85 promoter fragments from H1299 cells expressing a deletion mutant Del 491-155 (Figure 4a), which is incapable of Akt phosphorylation (Supplementary Figure S7). These results suggest that MDM2 or its deletion mutant Del 1-120 inhibits localization of REST on the p85 promoter, whereas the deletion mutant Del 491-155 does not.

We next determined if MDM2 or its deletion mutants Del 1120 and Del $491-155$ are capable of inhibiting the expression of p85 transcripts or proteins in H1299 cells. Our data revealed that the levels of both p85 transcripts (Figure $4 b$ ) and protein (Figure 4c) was downregulated in the H1299 constructs expressing MDM2 or Del 1-120, whereas Del 491155 did not show any reduction of p85 transcript or protein levels. Our observation signifies that MDM2 may dominantly interfere with the tumor suppressor function of the transcription factor REST to inhibit p85 expression and thus to upregulate Akt phosphorylation. Consistent with this observation, silencing of REST abolished the ability of MDM2 to enhance Akt phosphorylation and downregulate p85 expression (Supplementary Figure S8).

MDM2 reduces cell death induced by chemotherapeutic drugs. Overexpression of MDM2 has been related to increased survival of cancer cells harboring WT p53 after treatment with chemotherapeutic drugs, presumably due to MDM2-mediated inactivation and degradation of p53 induced by the chemotherapeutic drugs. ${ }^{6,33}$ As our experimental data indicate that MDM2 is capable of inducing Akt phosphorylation in the presence or absence of WT p53, and the Akt pathway has been related to reduced sensitivity of cancer cells to chemotherapeutic drugs, ${ }^{11,34,35}$ we investigated if MDM2 overexpression influences the sensitivity of cancer cells to chemotherapeutic drugs in the absence of WT p53.

Equal numbers of p53-null H1299 cells stably expressing MDM2 or stably transfected with empty vector were either treated with etoposide or vehicle and their survival was analyzed by colony development assay as reported earlier. ${ }^{36}$ The data show that cells stably overexpressing MDM2 generated fivefold more colonies than vector-transfected cells after treatment with etoposide (Figure 5a, Supplementary Figure S9 and Supplementary Table 1). We further determined if MDM2 reduces cell death induced by etoposide by examining cleavage of poly-ADP ribose polymerase (PARP) using western blot analysis. Our data showed diminished PARP cleavage in $\mathrm{H} 1299$ cells expressing MDM2 (Figures 5b and $\mathrm{c}$ ). These results suggest that MDM2 reduces sensitivity of cancer cells to etoposide even in the absence of p53.

An inhibitor of Akt activation, SH-6, inhibits MDM2mediated Akt phosphorylation and ability of MDM2 to reduce cell death induced by etoposide. As MDM2 reduced sensitivity of p53-null H1299 cells to etoposide (Figure 5a), we determined if an inhibitor of Akt phosphorylation, SH-6 (Supplementary Figures S2B and C), would abrogate the ability of MDM2 to alter etoposide sensitivity. Vector or MDM2-expressing H1299 stable transfectants were pre-treated with $\mathrm{SH}-6^{37}$ or the vehicle (DMSO). Equal number of treated cells was analyzed for their sensitivity to etoposide by colony development, and Akt phosphorylation and MDM2 expression by western blotting. The results show 


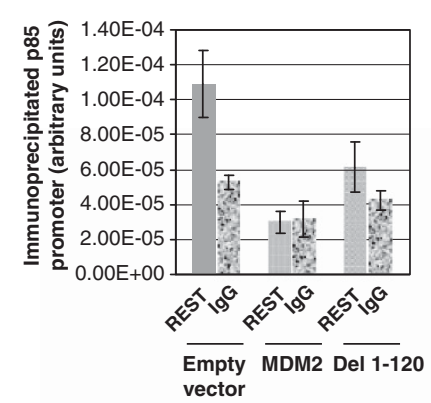

b

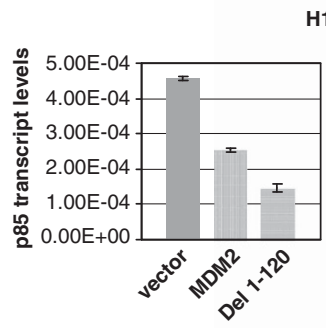

H1299

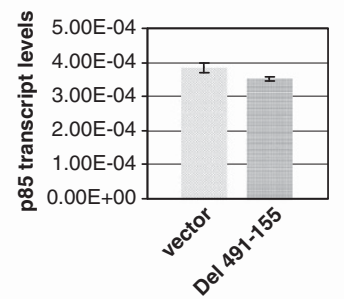

H1299

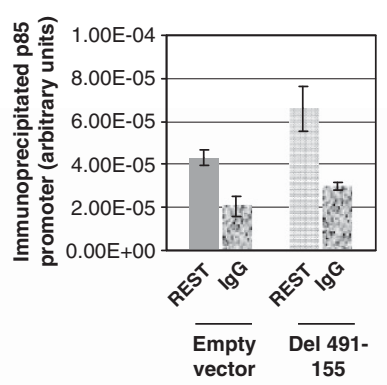

C

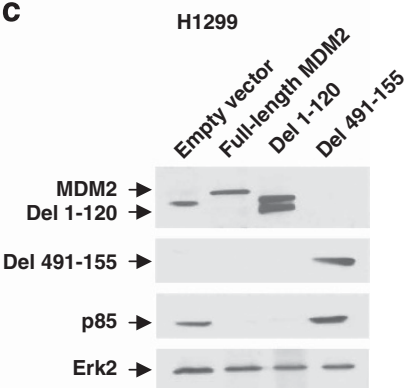

Figure 4 MDM2 inhibits localization of REST on the p85 promoter, and inhibits p85 expression. ChIP analysis of p85 promoter in extracts from $\mathrm{H} 1299$ cells expressing MDM2 or its deletion mutants Del 1-120 and Del 491-155 using a REST antibody (a). ChIP using IgG control is shown in each case. Expression of p85 transcript by QPCR analysis (b) and protein by western blot analysis (c) in H1299 cells expressing MDM2 or its deletion mutants are shown. Migration of MDM2 or its deletion mutants, p85 and Erk2 are indicated using arrows

a

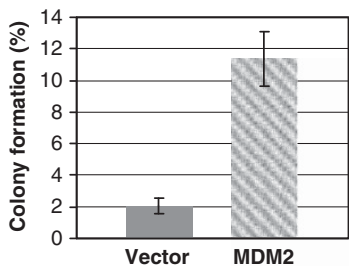

b

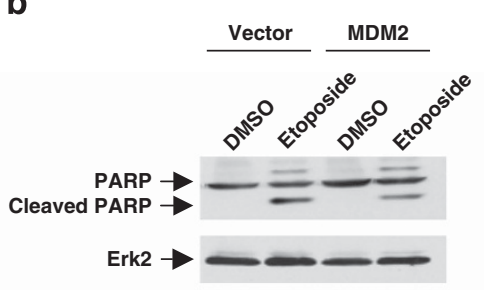

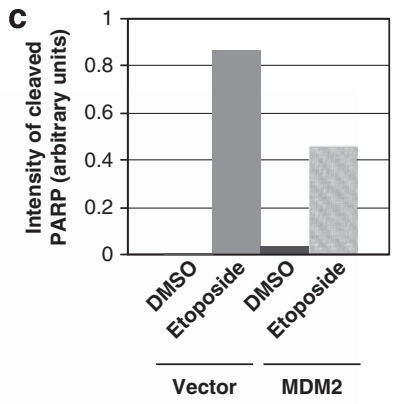

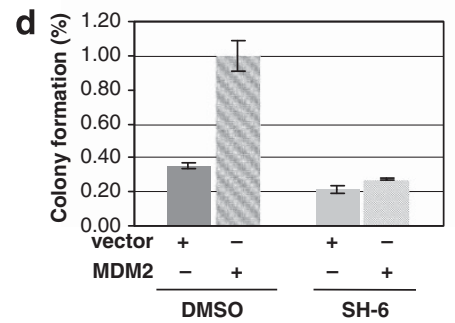

e

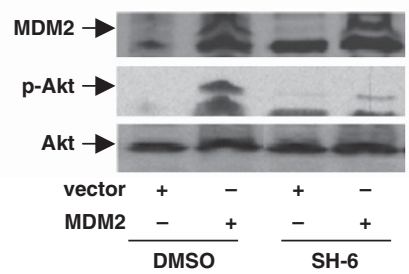

Figure 5 MDM2 reduces cell death induced by etoposide through its ability to phosphorylate Akt. Colony assays were performed after treating H1299 cells stably expressing MDM2 or stably transfected with vector plasmid with $1 \mu \mathrm{M}(\mathbf{a})$ or $6 \mu \mathrm{M}(\mathbf{b}, \mathbf{c})$ etoposide, and (d) Akt inhibitor, SH-6 (1.76 $\mu \mathrm{M})$ or DMSO followed by $6 \mu \mathrm{M}$ etoposide. Percent survival was calculated considering number of colonies generated in the control vehicle-treated plates to be $100 \%$. The results are shown by bar graphs (a and $\mathbf{d}$ ). Each experiment was performed in triplicate. Error bars are shown. PARP cleavage was determined by western blot analysis using PARP antibody (b). Migration of cleaved PARP and Erk2 are indicated. The band intensities of cleaved PARP normalized by PARP are shown by a bar graph (c). The lower right panel (e) shows inhibition of MDM2mediated Akt phosphorylation by the Akt inhibitor, SH-6 and MDM2 expression using western blot analysis

that while the MDM2-overexpressing H1299 stable transfectants pre-treated with DMSO generated approximately threefold more colonies than the corresponding vectortransfected cells after exposure to etoposide, pre-treatment with $\mathrm{SH}-6$ abrogated this ability of MDM2 (Figure $5 \mathrm{~d}$ and
Supplementary Table 2). SH-6 inhibited Akt phosphorylation in both the stable transfectants as expected (Figure 5e). These data show that the Akt phosphorylation function of MDM2 is required for its ability to increase cell survival after etoposide treatment. 
We have identified that amino-acid residues 121-275 of MDM2 are responsible for Akt phosphorylation (Supplementary Figure S7), and this domain of MDM2 is required for its ability to reduce cell death by etoposide (Supplementary Figure S7).

\section{Discussion}

The established model for oncogenic function of MDM2 states that MDM2 is phosphorylated by the Akt signaling pathway, which causes nuclear localization of the protein followed by its interaction with the tumor suppressor p53 and eventual degradation. Although this model is well evidenced in the literature, the incidence of MDM2 overexpression in cancers with mutant p53 is not infrequent, ${ }^{2}$ suggesting a WT p53independent role of MDM2 in conferring a survival advantage. In this communication, we present evidence to show a novel signaling pathway elicited by MDM2, where MDM2 interacts with the transcription factor REST, suppresses expression of the regulatory subunit of PI3-kinase and activates the Akt signaling pathway (Figure 6). This activation of the Akt pathway by MDM2 occurs both in normal and cancer cells in the presence or absence of p53 (Figure 1 and Supplementary Figures S1-4).

The novelty of this finding is that MDM2 physically and functionally interacts with the tumor suppressor REST, reported to downregulate PI3-kinase and Akt phosphorylation, and thus interfering with its ability to express p85 at the level of transcription. Although REST suppresses neural genes in non-neuronal cells, it also upregulates gene expression. $^{38}$ MDM2 may control expression of genes regulated by REST. Here we show how one of these incidences elicits a signaling pathway reducing cell death.

As Akt phosphorylates MDM2, MDM2-mediated Akt phosphorylation may constitute a positive feedback loop responsible for continuous transport of MDM2 to the nucleus. In normal cells, induction of p53 in response to DNA damage or other stress signals would induce MDM2 expression and therefore Akt phosphorylation perhaps to combat cell death. Overexpression of MDM2 in cancer cells would enhance this property and confer a survival advantage.

Recent literature reports that silencing of the tumor suppressor REST causes upregulation of PI3-kinase activity as well as Akt phosphorylation and cell transformation. ${ }^{14,39}$ Our data show that MDM2 prevents localization of REST on the p85 promoter, and induces an opposing function reducing the expression of p85 and increasing Akt phosphorylation (Figures 3 and 4 and Supplementary Figure S8). Interestingly, anti-MDM2 antibody did not co-precipitate the C terminally truncated REST4 in our immunoprecipitation assay, although A549 cells express this mutant (data not shown). This observation suggests that MDM2 may interact with the $\mathrm{C}$ terminus of REST to interfere with its function. ${ }^{40}$ We further show that silencing of REST lowers p85 expression; however, MDM2 did not increase Akt phosphorylation after knockdown of REST (Supplementary Figure S8). We often observed some decrease in Akt phosphorylation after REST knockdown in the presence of MDM2. As REST is an abundant DNA-bound protein, its knockdown may release bound MDM2, which then may degrade the p-Akt-bound proteins as reported earlier, destabilizing p-Akt. ${ }^{41}$

Interestingly, the PI3-kinase regulatory subunit p85 has been reported to exhibit a tumor suppressor function. ${ }^{29}$ Even a $20 \%$ decrease in its expression at the RNA level in human tumors has been correlated with the upregulation of Akt phosphorylation and tumor progression. ${ }^{29}$ Our data suggest that a $30 \%$ increase in the p85 transcript level generates enough p85 protein to inhibit Akt phosphorylation, whereas $50 \%$ reduction of p85 transcripts drastically inhibits p85 protein expression (Figures 1, 3 and 4). This observation indicates a p53-independent function of MDM2 in which MDM2 downregulates p85 expression to activate a prosurvival pathway (Figure 6). Interaction of MDM2 with REST also signifies a possible role for MDM2 in regulating transcription.

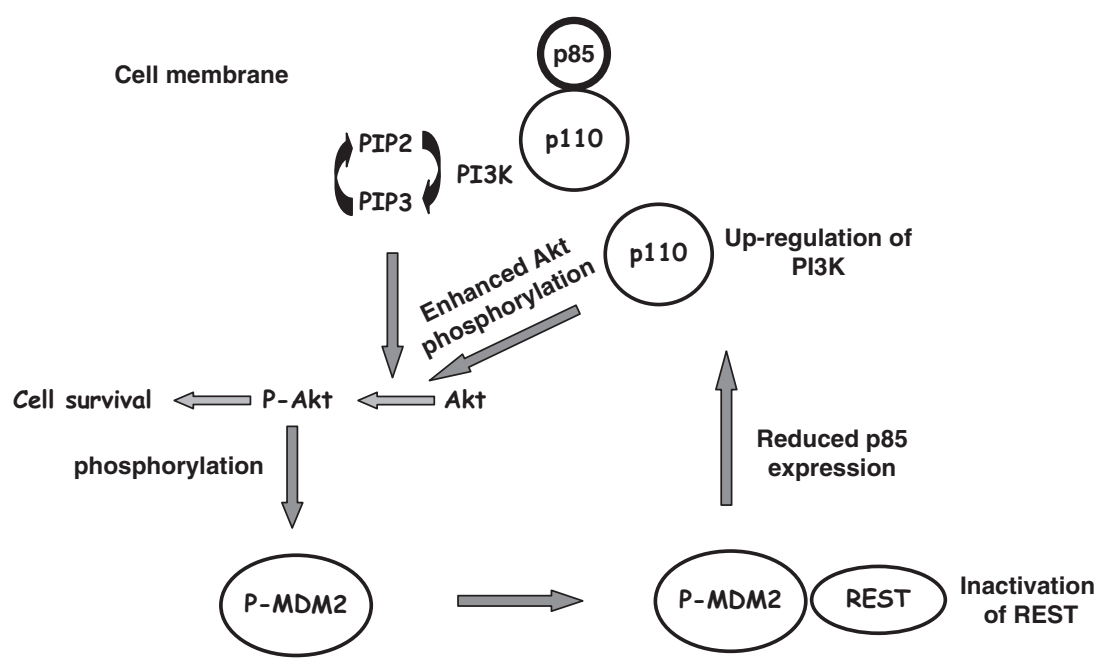

Nuclear localization

Cytoplasm

Nuclei

Figure 6 The cartoon proposes how MDM2 induces Akt phosphorylation as derived from our observation 
MDM2 harbors sequence elements found in transcription factors, ${ }^{1,3}$ although its target promoter elements are not predictable. Interaction of MDM2 with REST could explain its transcription regulatory function.

We show that MDM2 expression improves survival of cancer cells after treatment with etoposide in the absence of p53. We also show evidence that increased Akt phosphorylation by MDM2 reduced cell death (Figure 5). Consistently, mutants of MDM2 inactive in increasing Akt phosphorylation are also incapable of reducing cell death after treatment with etoposide, and a mutant that retains the ability of phosphorylating Akt retains this cell survival function (Supplementary Figure S7). Akt phosphorylation is known to enhance cell survival by various mechanisms. Our observation suggests a p53-independent mechanism for the prosurvival function of MDM2.

\section{Materials and Methods}

Plasmids, lentiviral vector and siRNA. Construction of plasmids expressing full-length MDM2 and its deletion mutants has been described in detail previously. ${ }^{42}$ Plasmids (pLKO.1) expressing shRNA against MDM2 or REST from the $\mathrm{U} 6$ promoter and harboring a puromycin resistance gene were purchased from Open Biosystems (Waltham, MA, USA). The control plasmid expresses shRNA against non-endogenous GFP gene. The lentiviral vectors to express MDM2 shRNA were generated by transfecting the recombinant plasmid encoding shRNA against MDM2 or non-endogenous GFP in 293T cells (American Type Culture Collection (ATCC), Manassas, VA, USA) by following the supplier's protocol. Akt and PI3-kinase activity reporters were gifts from Jin Zhang. ${ }^{22,23}$

Generation of MDM2 transgenic mice with p53 $+I+$ and p53 - Ibackground. To determine if MDM2 can elevate Akt phosphorylation, we generated MDM2 transgenic mice with a p53 $+/+$ and $p 53-/$ - background by cross-breeding p53 + / - /MDM2 transgene $+/$ - mice (gifts from Stephen Jones) and genotyped by analyzing DNA from tail biopsies as described. ${ }^{15}$

Cells, transfections and generation of stable transfectants. H1299, ABC1 and A549 cells were purchased from commercial sources, and were maintained in the media as suggested by the suppliers. OsACL cell line was a gift from AT Look. MEF cells from normal or p53 - / - /MDM2 - / - mice and tumor cells from p53-/-/MDM2 $-/-$ mice were gifts from Guillermina Lozano (Supplementary Information). We generated cultured lung cells from littermate MDM2 transgenic and non-transgenic mice with $\mathrm{p} 53+/+$, and $\mathrm{p} 53-/-$ background following a method described earlier. ${ }^{16}$ Methods for nucleofection, generation of MDM2 stable transfectants and lentiviral vectors were as described earlier. ${ }^{17}$ For nucleofection, cells were seeded 48 to $72 \mathrm{~h}$ before transfection. Cells were then trypsinized, and a suspension of $3 \times 10^{6}$ cells was mixed with plasmids or siRNA and pulsed (nucleofected) using a Nucleofector and kit reagents (Amaxa, Lonza, Allendale, NJ, USA) following the supplier's protocol and plated in 10-cm culture dishes in normal growth media. To generate MDM2 stable transfectants, H1299 cells were transfected with MDM2 expression plasmids (full-length or deletion mutants) or vector plasmid harboring neomycin resistance gene. To generate stable transfectants expressing shRNA against MDM2, REST or GFP appropriate cells were infected with lentiviral vectors expressing respective shRNA, ${ }^{17}$ and selected with appropriate antibiotics and either pooled or individual colonies were selected after analysis.

Western blot analysis. Western blot analysis was performed as described previously. ${ }^{42}$

Antibodies. Antibodies against MDM2 were chosen depending on the available epitopes. Ab-1 (Calbiochem, Millipore, Billerica, MA, USA), and N-20 and SMP14 (Santa Cruz Biotechnology, Santa Cruz, CA, USA) were used following the supplier's protocol. Antibodies against REST (Millipore), GSK3 $\beta$, extracellular signal-regulated kinase 2 (Erk2), p-Erk, Akt (Santa Cruz), Akt phosphorylated at Ser473, GSK3 $\beta$ phosphorylated at Ser 9, PARP and p85 (Cell Signaling Technology Inc., Danvers, MA, USA) and $\beta$-actin (Sigma, St. Louis, MO, USA) were also used following the manufacturer's protocol. $2 \mathrm{~A} 10$ antibody was a gift from Arnold Levine.
Analysis of Akt phosphorylation and PI3-kinase activity using respective activity reporter constructs. To determine whether upregulation of Akt phosphorylation or PI3-kinase activity upregulates downstream Akt signaling, respective activity reporters were used. The Akt or PI3-kinase activity reporters ${ }^{22,23}$ are chimeric proteins consisting of a phosphoamino acidbinding domain and a consensus substrate for the relevant kinase, phospho-Akt (p-Akt) or PI3-kinase, sandwiched between two green fluorescent protein mutants, CFP and YFP, respectively, capable of fluorescence resonance energy transfer or FRET. Excitation of CFP by a laser of $458 \mathrm{~nm}$ leads to cyan emission at $475 \mathrm{~nm}$, whereas YFP requires excitation with $514 \mathrm{~nm}$ laser for yellow emission at $530 \mathrm{~nm}$. When the consensus substrate peptide sequence is phosphorylated, it binds with the phosphoamino acid-binding domain, induces a structural change, and brings CFP and YFP close to each other. When CFP and YFP are brought together, excitation of CFP with a laser of $458 \mathrm{~nm}$ also excites YFP leading to emission at 530 even in the absence of a $514 \mathrm{~nm}$ laser (Supplementary Figure S3).

For analyzing the reporter activities, cells were nucleofected using the relevant activity reporter and an MDM2 expression plasmid (PCMV-MDM2) or vector plasmid (pCMV). Cells were then seeded onto coverslips. Consequently, the cells were fixed, washed, air dried and mounted with Prolong Gold Antifade (Molecular Probes, Life Technologies, Grand Island, NY, USA) and analyzed under a confocal microscope (Zeiss) under $\times 40$ magnification using the LSM image browser software (Zeiss, Thornwood, NY, USA). All images were collected using an excitation laser of $458 \mathrm{~nm}$. CFP images were collected using a 462- to $483-\mathrm{nm}$ emission filter and FRET (YFP) images were collected using a 526- to 537-nm emission filter. A single-color CFP construct was used to minimize bleed through in the YFP channel. Laser strength, pinhole, detector gain and amplifier gain were fixed for all samples. Fold increase of YFP/CFP channel mean intensity ratio over background fluorescence (autofluorescence) is shown by bar graphs.

RNA extraction, generation of cDNA and QPCR. Total RNA was isolated from exponentially growing cultured cell lines using TRIzol reagent (Life Technologies, Invitrogen) following a protocol supplied by the manufacturer. cDNA was synthesized using the Thermoscript reverse transcription-PCR system (Invitrogen). QPCR was performed using a LightCycler System (Roche Diagnostics Corporation, Indianapolis, IN, USA). Primers were designed using the OLIGO 5 software (Molecular Biology Insights, Inc., Cascade, CO, USA) and were synthesized by Integrated DNA Technologies (Coralville, IA USA). Reactions were performed in triplicate utilizing SYBR green dye, which exhibits a higher fluorescence upon binding of double-stranded DNA. The methods have been described previously. ${ }^{17}$ Reactions were performed in triplicate. The QPCR primers used were as follows: (a) MDM2 - forward, 5'-TGGCGTGCCAAGCTTCTCTGT-3' and reverse, 5'-ACCTGAGTCCGATGATTCCTGCT-3'; (b) GAPDH - forward, 5'-GTCAACGGATTTGGTCGTATT-3' and reverse, 5'-GATCTCGCTCCTGGAA GATGG-3'; (c) human p85 - forward, 5'-TTGCGAGGGAAGCGAGATGGC-3' and reverse, 5'-TGCTGCACAAGGGAGGTGTGT-3'.

ChIP analysis. H1299 cells expressing an empty vector or MDM2 expression plasmids were used for ChIP. Cells were plated at $4.5 \times 10^{6}$ cells per $150 \mathrm{~mm}$ plate and incubated with $2 \%$ formaldehyde for $10 \mathrm{~min}$ at $37^{\circ} \mathrm{C}$ to crosslink proteinDNA complexes. Crosslinking was stopped by adding $200 \mathrm{mM}$ glycine for $10 \mathrm{~min}$ at room temperature; cells were washed twice with $1 \times$ phosphate-buffered saline, and harvested. Cell pellets were resuspended in lysis buffer supplemented with $1 \%$ protease inhibitors. Extracts were passed through a $27^{1 / 2} \mathrm{G}$ needle and DNA was sonicated to $500-2 \mathrm{~kb}$ fragments on ice for $25 \mathrm{~min}$. Extracts were centrifuged and the protein content determined. Protein content was normalized between samples and precleared for $1 \mathrm{~h}$ at $4{ }^{\circ} \mathrm{C}$ with protein $\mathrm{A}$ agarose saturated with bovine serum albumin and sheared salmon sperm DNA. Extracts were then immunoprecipitated overnight using antibodies directed against REST (Millipore) and IgG (Santa Cruz) for control. Protein complexes were collected using protein A agarose saturated with bovine serum albumin and sheared salmon sperm DNA and washed once with RIPA buffer ( $150 \mathrm{mM} \mathrm{NaCl}, 50 \mathrm{mM}$ Tris $(\mathrm{pH} 8), 0.1 \%$ SDS, $0.5 \%$ sodium deoxycholate, $1 \% \mathrm{NP}-40)$, one time with high salt buffer $(500 \mathrm{mM}$ $\mathrm{NaCl}, 50 \mathrm{mM}$ Tris (pH 8), $0.1 \%$ SDS, $1 \%$ NP-40), two times in LiCl buffer $(250 \mathrm{mM}$ $\mathrm{LiCl}, 50 \mathrm{mM}$ Tris (pH 8), $0.5 \%$ sodium deoxycholate, $1 \% \mathrm{NP}-40$ ) and two times in $1 \times$ TE. Protein complexes were eluted in fresh elution buffer $(20 \%$ SDS, $10 \mathrm{mM}$ DTT, $100 \mathrm{mM} \mathrm{NaHCO}$ ) and incubated at $65^{\circ} \mathrm{C}$ overnight to reverse crosslinking. Samples were ethanol precipitated and then resuspended in $1 \times \mathrm{TE}$ and incubated with RNase $A(10 \mathrm{mg} / \mathrm{ml})$ for $30 \mathrm{~min}$ at $37^{\circ} \mathrm{C}$ and proteinase $\mathrm{K}$ $(10 \mathrm{mg} / \mathrm{ml})$ for $1 \mathrm{~h}$ at $42^{\circ} \mathrm{C}$. DNA was phenol-chloroform extracted and ethanol 
precipitated with $10 \mu \mathrm{g}$ glycogen. Samples were resuspended in water and the analysis performed using QPCR. Primers used for ChIP QPCR were $A \times / 15-\mathrm{kb}$ away (control region): forward, $5^{\prime}$-CCTTGACTGAGGCTTTACCA- $3^{\prime}$ and reverse, 5'-TTTTCAAAGTGCACCGACAT-3'; and p85 ChIP: forward, 5'-GCGTCCGAC CACACATGCCA-3' and reverse, 5'-GGGGGCAGAGGGGAGGAGTG-3'.

Inhibitors, chemotherapeutic drugs and IGF-1 treatment. Nutlin-3 (Sigma) treatment was carried out at $10 \mu \mathrm{M}^{6}$ for $24 \mathrm{~h}$. Wortmannin (Upstate Biotechnology Inc., Lake Placid, NY, USA) treatments were carried out at a concentration of $50 \mathrm{nM}$ for $4 \mathrm{~h}^{24}$ Akt inhibitor SH-6 (Calbiochem) was used at a concentration of $1.76 \mu \mathrm{M}$ for $4 \mathrm{~h}$. After pre-treatment, cells were either harvested for western blot analysis or plated to determine drug sensitivity by colony formation assay. Etoposide was purchased from Sigma.

Determination of drug sensitivity by colony formation assay. Colony formation assay was performed as reported earlier. ${ }^{36}$ Cells were seeded at equal density for vector-transfected and MDM2-expressing $\mathrm{H} 1299$ cells in $10-\mathrm{cm}$ plates in triplicate and treated with chemotherapeutic drugs at the specified concentration or the vehicle for $48 \mathrm{~h}$. Cells were then grown in normal growth media to allow colony formation by viable cells. After generation of colonies, cells were fixed with methanol, stained with methylene blue and colonies were counted. Percent survival of drug-treated vector-transfected or MDM2-expressing cells was calculated considering number of colonies generated in the respective control (DMSO)-treated plates to be $100 \%$. Each experiment was performed in triplicate.

Acknowledgements. We thank Dr. Arnold Levine for the monoclonal antibody 2A10, Guillermina Lozano for MEF cells from normal and p53 - / - /MDM2 - / mice, Stephen Jones for MDM2 transgenic mice and A.T. Look for OsACL cells. The Akt and PI3-kinase activity reporters are gifts from Jin Zhang. This study was initiated by a pilot project fund from the VCU Massey Cancer Center, and was supported by funds from Jeffress Memorial Trust to SPD and NCl (CA121144) to S.D.

1. Iwakuma T, Lozano G. MDM2, an introduction. Mol Cancer Res 2003; 1: 993-1000.

2. Onel K, Cordon-Cardo C. MDM2 and prognosis. Mol Cancer Res 2004; 2: 1-8.

3. Deb SP. Function and dysfunction of the human oncoprotein MDM2. Front Biosci 2002; 7 : d235-d243.

4. Bond GL, Hu W, Levine AJ. MDM2 is a central node in the p53 pathway: 12 years and counting. Curr Cancer Drug Targets 2005; 5: 3-8.

5. Michael D, Oren M. The p53-Mdm2 module and the ubiquitin system. Semin Cancer Biol 2003; 13: 49-58.

6. Vassilev LT, Vu BT, Graves B, Carvajal D, Podlaski F, Filipovic Z et al. In vivo activation of the p53 pathway by small-molecule antagonists of MDM2. Science 2004; 303: 844-848.

7. Rayburn E, Zhang R, He J, Wang H. MDM2 and human malignancies: expression, clinical pathology, prognostic markers, and implications for chemotherapy. Curr Cancer Drug Targets 2005; 5: 27-41.

8. Ogawara Y, Kishishita S, Obata T, Isazawa Y, Suzuki T, Tanaka K et al. Akt enhances Mdm2mediated ubiquitination and degradation of p53. J Biol Chem 2002; 277: 21843-21850.

9. Mayo LD, Donner DB. A phosphatidylinositol 3-kinase/Akt pathway promotes translocation of Mdm2 from the cytoplasm to the nucleus. Proc Natl Acad Sci USA 2001; 98 : 11598-11603

10. Frum R, Busby SA, Ramamoorthy M, Deb S, Shabanowitz J, Hunt DF et al. HDM2-binding partners: interaction with translation elongation factor EF1alpha. J Proteome Res 2007; 6 : 1410-1417.

11. Fresno Vara JA, Casado E, de Castro J, Cejas P, Belda-Iniesta C, Gonzalez-Baron M. PI3K/Akt signalling pathway and cancer. Cancer Treat Rev 2004; 30: 193-204.

12. Steelman LS, Chappell WH, Abrams SL, Kempf RC, Long J, Laidler $P$ et al. Roles of the Raf/MEK/ERK and PI3K/PTEN/Akt/mTOR pathways in controlling growth and sensitivity to therapy - implications for cancer and aging. Aging (Albany NY) 2011; 3: 192-222.

13. Keniry M, Parsons $R$. The role of PTEN signaling perturbations in cancer and in targeted therapy. Oncogene 2008; 27: 5477-5485.

14. Westbrook TF, Martin ES, Schlabach MR, Leng $Y$, Liang AC, Feng $B$ et al. A genetic screen for candidate tumor suppressors identifies REST. Cell 2005; 121: 837-848.

15. Jones SN, Hancock AR, Vogel H, Donehower LA, Bradley A. Overexpression of Mdm2 in mice reveals a p53-independent role for Mdm2 in tumorigenesis. Proc Natl Acad Sci USA 1998; 95: 15608-15612.
16. Parkinson EK, Yeudall WA. Epidermis. In: Masters JRW (ed) Human Cancer in Primary Culture: A Handbook. Kluwer Academic Publishers: Dordrecht, pp 187-197, 1991.

17. Frum R, Ramamoorthy M, Mohanraj L, Deb S, Deb SP. MDM2 controls the timely expression of cyclin A to regulate the cell cycle. Mol Cancer Res 2009; 7: 1253-1267.

18. Oliner JD, Kinzler KW, Meltzer PS, George DL, Vogelstein B. Amplification of a gene encoding a p53-associated protein in human sarcomas. Nature 1992; 358: 80-83.

19. Miwa S, Uchida C, Kitagawa K, Hattori T, Oda T, Sugimura H et al. Mdm2-mediated pRB downregulation is involved in carcinogenesis in a p53-independent manner. Biochem Biophys Res Commun 2006; 340: 54-61.

20. Vaughan C, Mohanraj L, Singh S, Dumur Cl, Ramamoorthy M, Garrett CT et al. Human oncoprotein MDM2 up-regulates expression of NF-kappaB2 precursor p100 conferring a survival advantage to lung cells. Genes Cancer 2011; 2: 943-955.

21. Ikediobi ON, Davies H, Bignell G, Edkins S, Stevens C, O'Meara $S$ et al. Mutation analysis of 24 known cancer genes in the NCl-60 cell line set. Mol Cancer Ther 2006; 5 : 2606-2612.

22. Ananthanarayanan $B, N i \mathrm{Q}$, Zhang J. Signal propagation from membrane messengers to nuclear effectors revealed by reporters of phosphoinositide dynamics and Akt activity. Proc Natl Acad Sci USA 2005; 102: 15081-15086.

23. Gao X, Zhang J. Spatiotemporal analysis of differential Akt regulation in plasma membrane microdomains. Mol Biol Cell 2008; 19: 4366-4373.

24. Thelen M, Wymann MP, Langen H. Wortmannin binds specifically to 1-phosphatidylinositol 3-kinase while inhibiting guanine nucleotide-binding protein-coupled receptor signaling in neutrophil leukocytes. Proc Natl Acad Sci USA 1994; 91: 4960-4964.

25. Zhao L, Vogt PK, Class I. PI3K in oncogenic cellular transformation. Oncogene 2008; 27: 5486-5496.

26. Backer JM. The regulation of class IA PI 3-kinases by inter-subunit interactions. Curr Top Microbiol Immunol 2010; 346: 87-114.

27. Jaiswal BS, Janakiraman V, Kljavin NM, Chaudhuri S, Stern HM, Wang W et al. Somatic mutations in p85alpha promote tumorigenesis through class IA PI3K activation. Cancer Cell 2009; 16: 463-474.

28. Sun M, Hillmann P, Hofmann BT, Hart JR, Vogt PK. Cancer-derived mutations in the regulatory subunit p85alpha of phosphoinositide 3-kinase function through the catalytic subunit p110alpha. Proc Natl Acad Sci USA 2010; 107: 15547-15552.

29. Taniguchi CM, Winnay J, Kondo T, Bronson RT, Guimaraes AR, Aleman JO et al. The phosphoinositide 3-kinase regulatory subunit p85alpha can exert tumor suppressor properties through negative regulation of growth factor signaling. Cancer Res 2010; 70: 5305-5315.

30. Sarbassov DD, Guertin DA, Ali SM, Sabatini DM. Phosphorylation and regulation of Akt/ PKB by the rictor-mTOR complex. Science 2005; 307: 1098-1101.

31. Coulson JM. Transcriptional regulation: cancer, neurons and the REST. Curr Biol 2005; 15: R665-R668.

32. Majumder S. REST in good times and bad: roles in tumor suppressor and oncogenic activities. Cell Cycle 2006; 5: 1929-1935.

33. Liu TG, Yin JQ, Shang BY, Min Z, He HW, Jiang JM et al. Silencing of hdm2 oncogene by siRNA inhibits p53-dependent human breast cancer. Cancer Gene Ther 2004; 11: 748-756.

34. Kim SH, Juhnn YS, Song YS. Akt involvement in paclitaxel chemoresistance of human ovarian cancer cells. Ann NY Acad Sci 2007; 1095: 82-89.

35. Yu HG, Ai YW, Yu LL, Zhou XD, Liu J, Li JH et al. Phosphoinositide 3-kinase/Akt pathway plays an important role in chemoresistance of gastric cancer cells against etoposide and doxorubicin induced cell death. Int $J$ Cancer 2008; 122: 433-443.

36. Scian MJ, Stagliano KE, Anderson MA, Hassan S, Bowman M, Miles MF et al. Tumor-derived p53 mutants induce NF-kappaB2 gene expression. Mol Cell Biol 2005; 25: 10097-10110.

37. Kozikowski AP, Sun H, Brognard J, Dennis PA. Novel PI analogues selectively block activation of the pro-survival serine/threonine kinase Akt. J Am Chem Soc 2003; 125: 1144-1145.

38. Qureshi IA, Gokhan S, Mehler MF. REST and CoREST are transcriptional and epigenetic regulators of seminal neural fate decisions. Cell Cycle 2010; 9: 4477-4486.

39. Zhao JJ, Gjoerup OV, Subramanian RR, Cheng Y, Chen W, Roberts TM et al. Human mammary epithelial cell transformation through the activation of phosphatidylinositol 3-kinase. Cancer Cell 2003; 3: 483-495.

40. Lietz M, Bach K, Thiel G. Biological activity of RE-1 silencing transcription factor (REST) towards distinct transcriptional activators. Eur J Neurosci 2001; 14: 1303-1312.

41. Gama V, Gomez JA, Mayo LD, Jackson MW, Danielpour D, Song K et al. Hdm2 is a ubiquitin ligase of Ku70-Akt promotes cell survival by inhibiting Hdm2-dependent Ku70 destabilization. Cell Death Differ 2009; 16: 758-769.

42. Leng P, Brown DR, Shivakumar CV, Deb S, Deb SP. N-terminal 130 amino acids of MDM2 are sufficient to inhibit p53-mediated transcriptional activation. Oncogene 1995; 10: $1275-1282$. 